\title{
Cancer Cells with Stem Cell-Like Phenotypes and Liver Metastasis from Colon Carcinoma
}

\author{
Antonia Bellizzi and Stefania Tommasi* \\ National Cancer Research Centre, Istituto Tumori "Giovanni Paolo II", Bari, Italy
}

\begin{abstract}
Tumor heterogeneity contributes to the potentiality of tumors to invade and metastasize. Thus the identification of specific cells programmed to go to a specific site and establish themselves, actively proliferating and causing metastasis, could be fundamental for early diagnosis and for selective targeted therapy. Apart from gene signatures which seem to identify this tumor ability, selected cell populations with self-renewal potential have been recognized: stem cell-like cancer cells. In this paper we review recent evidence on the identification of colon cancer cells with stem cell-like properties which could have a key role in the liver metastasization. The potential clinical implication of their therapeutic eradication is also discussed.
\end{abstract}

Keywords: Stem Cell-Like Cancer cells (SCLC); Colon cancer; Metastasis

Colorectal Cancer (CRC) is the third most common cancer in men (663,000 cases, $10.0 \%$ of the total) and the second in women $(570,000$ cases, $9.4 \%$ of the total) worldwide. Almost $60 \%$ of the cases occur in developed regions [1] and approximately $50 \%$ of patients develop synchronous or metachronous liver metastases within 2 years from the resection of the primary tumor [2]. Most colon cancer deaths result from the metastatic spread of tumor cells to the liver and other organs [3] and for this reason it is of great significance to identify new markers capable of discriminating earlier the potential that a primary tumor has to initiate a process of liver metastasization. The standard care in patients with operable liver-confined lesions is represented by a combination of chemotherapy and surgery [4]. However, there are still some critical issues to be solved. Firstly, surgical cures are relatively rare in this setting. Secondly, how to juxtapose chemotherapy and surgery, and the duration of chemotherapy. Finally, the possibility of pre-operative chemotherapy in the case of resectable metastases [5]. This is primarily due to the fact that current chemotherapy attacks the bulk of the cancer without affecting the chemo-resistant Stem Cell-Like Cancer cells (SCLCs). These can re-grow after treatment and, eventually, develop the changes responsible for the occurrence of drug-resistance [6-8] and facilitate a persistent and aggressive tumor phenotype, capable of invading other sites. In this scenario, understanding what drives metastasization to the liver and the characterization of clonal cells which selectively establish themselves in that site acquires great importance.

In CRC, SCLCs are colon stem cells progenitors whose deregulated dynamics lead to the origin of a tumor [9]. At least two populations of progenitors are identified at the bottom of the normal colon Lieberkühn: true quiescent stem cells with high chemoresistance, and low proliferating potential [10]. They produce continuously the second compartment of stem cell progenitors with high mitogenic, differentiation and migratory potentials towards the top epithelium surface of the crypts. Upon migration upward through the crypt, these cells proliferate and differentiate into one of the fourth epithelial cell types of the crypt wall, constituting the single sheet epithelial layer folded into finger-like invaginations [11]. These two stem and progenitor populations are the real targets of the neoplastic transformation. Due to their long survival they acquire multiple genetic mutations leading to carcinogenesis. Furthermore, they have the ability to regenerate and exhibit resistance to conventional therapies, useless for quiescent cells with asymmetrical division [12]. On the same grounds stem cells are ideal candidates for the transformation events that occur in metastatic disease.

The majority of patients with CRC develop metastases principally within two target organs: lung and liver [13]. There are growing evidence pointing to chemokine involvement in these organ-selective metastases $[14,15]$, and recently, a specific role of the CXCR7/chemokine axis has been demonstrated in lung compared to liver metastases [16]. However, the differential tropism from CRC to several target sites is still not well explained, including the mechanisms that drive hepatic homing. There have been a few studies performed on liver colonization by metastatic cells [17], on the modulation of pharmacological response by the liver microenvironment [18], and on the existence of peptide ligands specific for liver metastasis [19]. However, there have been no studies in this direction involving SCLCs. Indeed, current therapies for advanced CRC target molecules related to cancer progression (e.g. VEGF and EGFR) [20,21], not considering factors/cells involved in the initiation of metastasis.

In previous literature, different markers have been used to identify SCLCs and, among these, CD133, CD166 and EpCAM resulted to be well adapted to the versatility and multiplicity of the phenotypes attributed to the SCLC. CD133, originally known as AC133, is a glycoprotein also known in humans and rodents as Prominin 1 (PROM1) [22]. It is the founding member of the prominin family of pentaspan transmembrane glycoproteins. The specific functions and ligands of prominins are still unclear, but they are expressed within plasma membrane protrusions, such as epithelial microvilli and epididymal ductal epithelial stereocilia [23]. CD133 was specifically regarded as the colon cancer stem cell-surface marker and has been

${ }^{*}$ Corresponding author: Stefania Tommasi, Molecular Genetics Laboratory, National Cancer Research Centre, Istituto Tumori "Giovann Paolo II", v.le Orazio Flacco 65, 70124 Bari, Italy, Tel: 0039-0805555283; E-mail: s.tommasi@oncologico.bari.it

Received October 31, 2012; Accepted December 21, 2012; Published December 28, 2012

Citation: Bellizzi A, Tommasi S (2013) Cancer Cells with Stem Cell-Like Phenotypes and Liver Metastasis from Colon Carcinoma. J Liver 2: 114 doi:10.4172/2167-0889.1000114

Copyright: () 2013 Bellizzi A, et al. This is an open-access article distributed under the terms of the Creative Commons Attribution License, which permits unrestricted use, distribution, and reproduction in any medium, provided the original author and source are credited. 
Citation: Bellizzi A, Tommasi S (2013) Cancer Cells with Stem Cell-Like Phenotypes and Liver Metastasis from Colon Carcinoma. J Liver 2: 114. doi:10.4172/2167-0889.1000114

Page 2 of 5

used to identify and isolate cancer initiating cells from human colon cancer [24]. It has been demonstrated that CD133+ cells are able to maintain themselves as well as differentiate and re-establish tumor heterogeneity upon serial transplantation in vivo [25-27].

ALCAM/CD166 is a glycoprotein that is involved in both homotypic and heterotypic (to lymphocyte cell-surface receptor CD6) adhesion and is over-expressed in several tumors [28], including colon cancer [29]. In human CRC, aberrant cell surface CD166 expression is strongly correlated with a 15 month shortened survival [29]. CD166 cell surface expression was found in the endogenous intestinal stem cell niche, suggesting that as a cell adhesion molecule, it may play an important role in maintaining the integrity of the stem cell niche, or in directing cells to the crypt base [30]. EpCAM is a glycosylated, $30-40 \mathrm{kDa}$ type I membrane protein, which is expressed in a variety of human epithelial tissue cancers, as well as normal progenitor and stem [31]. It is expressed in all human adenocarcinomas, squamous cell carcinomas, retinoblastomas and hepatocellular carcinomas [32]. The correlation of EpCAM expression and poor survival has been described in several tumor types, including invasive breast cancer [33], urothelial carcinoma of the bladder [34], gallbladder carcinoma [35] and squamous cell carcinoma of the oesophagus [36].

Moreover, a CD44-positive subpopulation, with tumorigenic and self-renewal potential, has been selected in MCF7 breast cancer cellengineered mammospheres [37]. CD44 is a cell surface glycoprotein involved in cell-cell interaction, cell adhesion and migration. It was identified as the cell-surface marker of breast cancer and also recently observed to be related to the distant metastasis of colon cancer [38]. CD44 exists as a large family of isoforms, produced by the alternative splicing of up to 20 exons, which generate different binding sites for the molecule $[39,40]$. These variant isoforms are expressed in many different organs and have been strongly linked to tumor progression behaviors in various cancers [41-43]. Expression of CD44 is widely identified in cancer stem cells in various organs, such as breast, colon, and pancreas [44-46].

Among these SCLC markers, CD133 and CD44, already validated as informative markers of stem cells in both primary tumors and xenografts, $[24,25]$ have been identified in gene expression signatures whose genes have been shown to enhance cell migration and invasion activity from colon cancer to liver metastasis [47].

Our own study [48] evidenced a particular group of cells able to specifically metastasize to the liver which express CD133/CD44. To achieve these results, human colonosphere cultures of stem cells were obtained from fresh samples deriving from colon cancer and liver metastasis specimens under serum-free culture conditions. Phenotypical and functional characteristics of SCLCs were compared to those present in the corresponding non tumor counterpart, adjacent to the neoplastic lesion. The expression of CD133 and CD44 in our own non-selected naïve samples (Figure 1), in clones obtained after 5 months of cell selection (Figure 2) and in tissue slices of the same patient demonstrated that a CD133+/CD44+ population was present in primary CRCs and in metastatic liver tumors. Furthermore, the amount of CD133+/CD44+ SCLCs in metastatic liver tumors was greater than that in primary colorectal tumors, while CD133+/CD44+ SCLCs were hardly detected in the corresponding normal colorectal tissue. These data demonstrate an enrichment of CD133+CD44+ cells in metastatic cancers, which underlines that these cells may play a potential role in hepatic driving metastasis of CRC.

Recently, in line with this, there have been some studies addressing
CD133 and CD44 co-expression as cancer stem cell antigens in colon cancer patients $[49,50]$. CD44/CD133 co-expression was significantly higher in colon cancers with early liver metastases compared to those without, suggesting that CD133 and CD44 protein co-expression in colon cancer may be a potential biomarker for early liver metastasization [38]. Indeed, Chen et al. demonstrated that sorted CD133+/CD44+ cells are undifferentiated, endowed with extensive self-renewal and epithelial lineage differentiation capacity in vitro, more invasive in vitro and responsible solely for liver metastasis in vivo [51].

Recently Fang et al. evaluated miRNA expression differences between colon SCLCs with the CD133+/CD44+ and CD133-/CD44surface phenotype from the human SW1116 colon adenocarcinoma cell line, emphasizing that the difference between the two immunophenotypes was due to miRNAs closely related not only to cell cycle and cell differentiation pathways, keys to distinguish stem cells from non-stem cells, but also to signaling pathways related to metastatic behavior, such as cytoskeletal proteins and cell-matrix adhesion [52]. Moreover the combined CD133/CD44 expression seemed to be a strong indicator of worse disease-free survival and an independent risk factor for recurrence [53]. These data further confirm our findings on the presence of CD133+/CD44+ cells in all patients with liver metastasis, while only in $10 \%$ of non-metastatic patients [38]. All these data highlighted that CD133 and CD44 could be markers of metastasis-facilitating pathways through which liver tumor metastasis is jointly promoted.

Another key player in the biology of the metastatic process is represented by circulating tumor cells (CTCs) [54], and recent
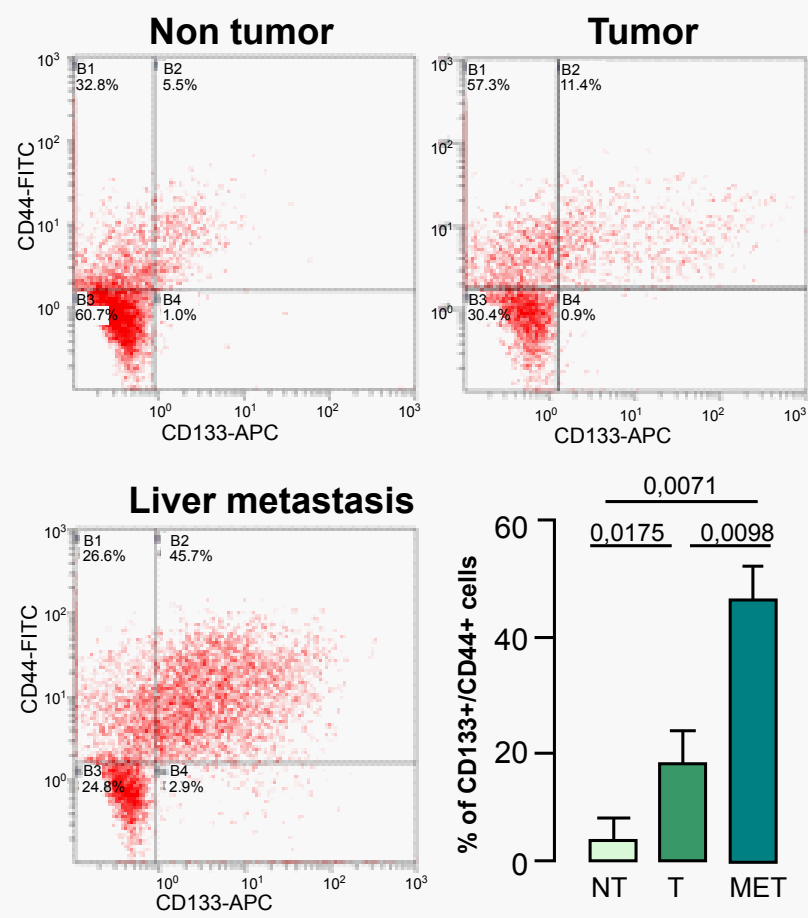

Figure 1: A representative image of CD133+CD44+ cell content in Non Tumor tissues (NT), primary colorectal tumors ( $T$ ) and liver Metastasis (MET). Specimens were digested, double-stained for CD133 and CD44 and analyzed by flow cytometry to detect the CD133+/CD44+ subpopulation. Quantification of flow cytometry data is presented down in the right panel $(n=8$ analyzed patients). Data were expressed as means \pm SD. Statistical analysis was performed by Student's $t$ test $(P<0.05)$. 
Citation: Bellizzi A, Tommasi S (2013) Cancer Cells with Stem Cell-Like Phenotypes and Liver Metastasis from Colon Carcinoma. J Liver 2: 114. doi:10.4172/2167-0889.1000114

Page 3 of 5

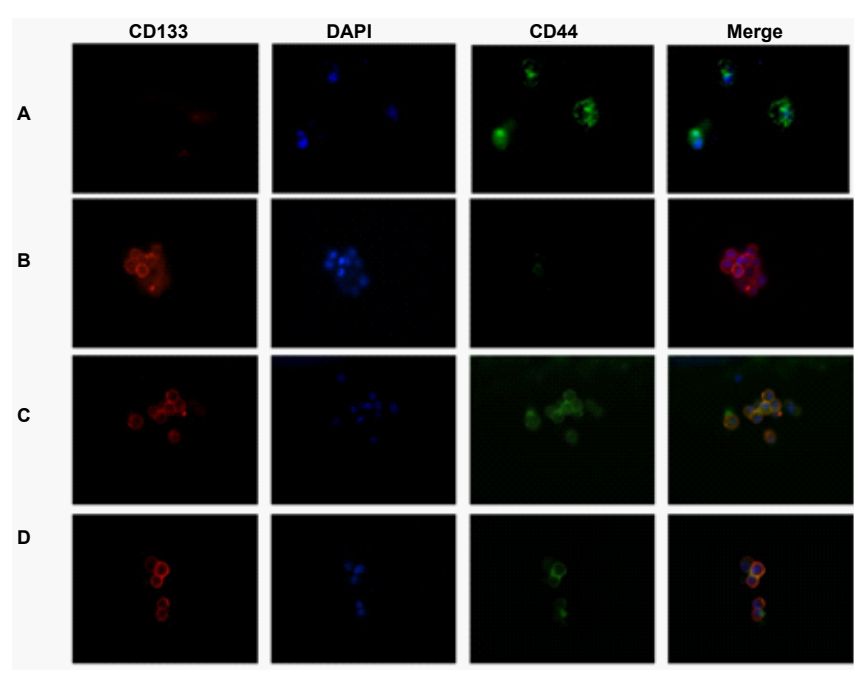

Figure 2: Representative images of CD44 and CD133 expression in colon sphere growth for 5 months under serum-free conditions. Tumorspheres were tripsinized, double-stained for CD133 and CD44 and analyzed by immunoflorescent technique to detect the CD133+/CD44+ subpopulation in SCLCs obtained from A) non tumor tissues, B) primary colorectal tumors of patients without liver metastasis (M0), C) primary colorectal tumors of patients with liver metastasis (M1) and D) liver metastasis. Images of single stained colonosphere with CD133 (red, first column) and CD44 (green third column) antibody, and double staining are shown (Merge). Nuclei have been evidenced by DAPI. SCLCs isolated from the non tumor compartments were all CD133-/CD44+; those isolated from M0 patients were CD133+/CD44, while those isolated from M1 patients were all CD133+/CD44+. (Modified from Bellizzi et al. [47]).

observations support the hypothesis that detection of putative CD133 circulating cancer stem cells in the peripheral blood of patients affected by CRC might identify high-risk patients and could be an indicator of disease progression after apparently radical surgery [52,55]. We also found a detectable population of CTCs in $75 \%$ of metastatic colon cancer patients: $20 \%$ of CTCs showed membrane CD133+/CD44+ accumulation, supporting the premises that CD133+/CD44+ CTCs represent a sampling of the phenotypic cell types present in the primary and metastatic tumor deposits, and the possibility that circulating tumor cells consist of a combination of cells with malignant potential, stem cell characteristics and actively migrating cells that may go on to form liver metastatic foci.

\section{Influence of SCLCs in the Therapeutic Setting}

Interestingly, CD133 and CD44 have already been evaluated together or individually as cell surface markers of resistance to conventional therapies utilized in CRC: 5-Fluorouracil (5FU), oxaliplatin and irinotecan. In particular it has been demonstrated that $\mathrm{CD} 133+/ \mathrm{CD} 44+$ colorectal cancer cells give rise to long-term tumor sphere (or spheroids) more resistant to the chemotherapeutic irinotecan [56], and that 5-fluorouracil (FU) resistant spheroid clones adopted a CD133+ CD44- phenotype [57-59].

Recent studies have demonstrated how it is already possible to modulate proliferation of tumorspheres and drug resistance in this particular population. Reduction of mRNA and protein levels of CD44 and CD133 in both FOLFOX-resistant/non resistant colon cancer cell lines could be for example triggered by the activation of the gene schlafen-3 (Slfn-3), whose expression correlates with intestinal epithelial cell differentiation [60]. This resulted in the inhibition of multiple characteristics of SCLC-enriched, FOLFOX-resistant colon cancer cells, such as decreased formation of tumorsphere in stem cell medium, inhibition of expression of the chemotherapeutic drug transporter protein ABCG2, and a reduced Hoechst 33342 dye exclusion. Thus Slfn-3 expression may render CD44+/CD133+ colon SCLCs more susceptible to cancer chemotherapeutics [61].

Moreover, by blocking endogenous miR-140, significantly elevated in $\mathrm{CD} 133+/ \mathrm{CD} 44+$ colon cancer cells, it could be possible to partially sensitize resistant colon cancer cells to 5-FU treatment [56]. SB-T-1214, one of the new-generation taxoids which was developed as an attempt to improve widely used taxane-based anticancer agents [62], has been demonstrated to induce growth inhibition and apoptotic cell death in drug resistant $[63,64]$ and tumorigenic CD133+/CD44+ colon cancer spheroids [65], suggesting that this compound can specifically target tumor-specific SCLCs by inhibiting some stemness-related signaling pathways.

\section{Conclusions}

The identification of SCLCs expressing CD133/CD44 as a potential marker of hepatic metastasization has importance for both diagnostic as well as therapeutic purposes. Targeting SCLC by these surface markers could be a valid approach to selectively eliminate SCLC that is able to metastasize to liver. Furthermore, these studies highlighted how the identified cell population could be responsible for the pharmacological resistance to conventional and new therapies, but it is already possible to identify the elements within it which may become reasonable targets for an effective drug therapy.

\section{Acknowledgements}

The authors would like to thank Dr. Caroline Oakley for language revision.

\section{References}

1. Ferlay J, Shin HR, Bray F, Forman D, Mathers C, et al. (2010) Estimates of worldwide burden of cancer in 2008: GLOBOCAN 2008. Int J Cancer 127: 2893-2917.

2. Parkin DM, Bray F, Ferlay J, Pisani P (2005) Global cancer statistics, 2002. CA Cancer J Clin 55: 74-108.

3. Zhang SS, Han ZP, Jing YY, Tao SF, Li TJ, et al. (2012) CD133(+)CXCR4(+) colon cancer cells exhibit metastatic potential and predict poor prognosis of patients. BMC Med 10: 85.

4. Tomlinson JS, Jarnagin WR, DeMatteo RP, Fong Y, Kornprat P, et al. (2007) Actual 10-year survival after resection of colorectal liver metastases defines cure. J Clin Oncol 25: 4575-4580.

5. Nordlinger B, Vauthey JN, Poston G, Benoist S, Rougier P, et al. (2010) The timing of chemotherapy and surgery for the treatment of colorectal liver metastases. Clin Colorectal Cancer 9: 212-218.

6. Guan Y, Gerhard B, Hogge DE (2003) Detection, isolation, and stimulation of quiescent primitive leukemic progenitor cells from patients with acute myeloid leukemia (AML). Blood 101: 3142-3149.

7. Guzman ML, Swiderski CF, Howard DS, Grimes BA, Rossi RM, et al. (2002) Preferential induction of apoptosis for primary human leukemic stem cells. Proc Natl Acad Sci U S A 99: 16220-16225

8. Dean M, Fojo T, Bates S (2005) Tumour stem cells and drug resistance. Nat Rev Cancer 5: 275-284.

9. Sipos F, Valcz G, Molnár B (2012) Physiological and pathological role of loca and immigrating colonic stem cells. World J Gastroenterol 18: 295-301.

10. Gespach C (2010) Stem cells and colon cancer: the questionable cancer stem cell hypothesis. Gastroenterol Clin Biol 34: 653-661.

11. Nautiyal J, Kanwar SS, Yu Y, Majumdar AP (2011) Combination of dasatinib and curcumin eliminates chemo-resistant colon cancer cells. J Mol Signal 6: 7.

12. Yen TH, Wright NA (2006) The gastrointestinal tract stem cell niche. Stem Cel Rev 2: 203-212. 
Citation: Bellizzi A, Tommasi S (2013) Cancer Cells with Stem Cell-Like Phenotypes and Liver Metastasis from Colon Carcinoma. J Liver 2: 114. doi:10.4172/2167-0889.1000114

13. Weitz J, Koch M, Debus J, Höhler T, Galle PR, et al. (2005) Colorectal cancer. Lancet 365: 153-165

14. Zlotnik A (2008) New insights on the role of CXCR4 in cancer metastasis. J Pathol 215: 211-213

15. Mukaida N, Baba T (2012) Chemokines in tumor development and progression. Exp Cell Res 318: 95-102.

16. Guillemot E, Karimdjee-Soilihi B, Pradelli E, Benchetrit M, Goguet-Surmenian E, et al. (2012) CXCR7 receptors facilitate the progression of colon carcinoma within lung not within liver. Br J Cancer 107: 1944-1949.

17. Kuo TH, Kubota T, Watanabe M, Furukawa T, Teramoto T, et al. (1995) Live colonization competence governs colon cancer metastasis. Proc Natl Acad Sci USA 92: 12085-12089.

18. Dong Z, Radinsky R, Fan D, Tsan R, Bucana CD, et al. (1994) Organ-specific modulation of steady-state mdr gene expression and drug resistance in murine colon cancer cells. J Natl Cancer Inst 86: 913-920.

19. Marchiò S, Soster M, Cardaci S, Muratore A, Bartolini A, et al. (2012) A complex of a6 integrin and E-cadherin drives liver metastasis of colorectal cancer cells through hepatic angiopoietin-like 6. EMBO Mol Med 16 4: 1156-1175.

20. Grothey A, Galanis E (2009) Targeting angiogenesis: progress with anti-VEGF treatment with large molecules. Nat Rev Clin Oncol 6: 507-518.

21. Okamoto I (2010) Epidermal growth factor receptor in relation to tumor development: EGFR-targeted anticancer therapy. FEBS J 277: 309-215.

22. Kawamoto H, Yuasa T, Kubota Y, Seita M, Sasamoto H, et al. (2010) Characteristics of CD133(+) human colon cancer SW620 cells. Cell Transplant 19: 857-864

23. Mizrak D, Brittan M, Alison M (2008) CD133: molecule of the moment. JPatho 214: 3-9

24. Ricci-Vitiani L, Lombardi DG, Pilozzi E, Biffoni M, Todaro M, et al. (2007) Identification and expansion of human colon-cancer-initiating cells. Nature 445 : 111-115.

25. O'Brien CA, Pollett A, Gallinger S, Dick JE (2007) A human colon cancer cell capable of initiating tumour growth in immunodeficient mice. Nature 445: 106110.

26. Yin S, Li J, Hu C, Chen X, Yao M, et al. (2007) CD133 positive hepatocellular carcinoma cells possess high capacity for tumorigenicity. Int J Cancer 120: $1444-1450$.

27. Suetsugu A, Nagaki M, Aoki H, Motohashi T, Kunisada T, et al. (2006) Characterization of CD133+ hepatocellular carcinoma cells as cancer stem/ progenitor cells. Biochem Biophys Res Commun 351: 820-824.

28. Ofori-Acquah SF, King JA (2008) Activated leukocyte cell adhesion molecule: a new paradox in cancer. Transl Res 151: 122-128.

29. Weichert W, Knösel T, Bellach J, Dietel M, Kristiansen G (2004) ALCAM/ CD166 is overexpressed in colorectal carcinoma and correlates with shortened patient survival. J Clin Pathol 57: 1160-1164.

30. Levin TG, Powell AE, Davies PS, Silk AD, Dismuke AD, et al. (2010) Characterization of the intestinal cancer stem cell marker CD166 in the human and mouse gastrointestinal tract. Gastroenterology 139: 2072-2082.

31. Gires O, Klein CA, Baeuerle PA (2009) On the abundance of EpCAM on cancer stem cells. Nat Rev Cancer 9: 143.

32. Baeuerle PA, Gires O (2007) EpCAM (CD326) finding its role in cancer. Br J Cancer 96: 417-423.

33. Spizzo G, Obrist P, Ensinger C, Theurl I, Dünser M, et al. (2002) Prognostic significance of Ep-CAM AND Her-2/neu overexpression in invasive breas cancer. Int J Cancer 98: 883-888.

34. Brunner A, Prelog M, Verdorfer I, Tzankov A, Mikuz G, et al. (2008) EpCAM is predominantly expressed in high grade and advanced stage urothelial carcinoma of the bladder. J Clin Pathol 61: 307-310.

35. Prince S, Zeidman A, Dekel Y, Ram E, Koren R (2008) Expression of epithelia cell adhesion molecule in gallbladder carcinoma and its correlation with clinicopathologic variables. Am J Clin Pathol 129: 424-429.

36. Stoecklein NH, Siegmund A, Scheunemann P, Luebke AM, Erbersdobler A, et al. (2006) Ep-CAM expression in squamous cell carcinoma of the esophagus: a potential therapeutic target and prognostic marker. BMC Cancer 6: 165.
37. Lee E, Hong Y, Choi J, Haam S, Suh JS, et al. (2012) Highly selective CD44 specific gold nanorods for photothermal ablation of tumorigenic subpopulations generated in MCF7 mammospheres. Nanotechnology 23: 465101.

38. Huang X, Sheng Y, Guan M (2012) Co-expression of stem cell genes CD133 and CD44 in colorectal cancers with early liver metastasis. Surg Oncol 21 103-107.

39. Goodison S, Urquidi V, Tarin D (1999) CD44 cell adhesion molecules. Mol Pathol 52: 189-196.

40. Naor D, Sionov RV, Ish-Shalom D (1997) CD44: structure, function, and association with the malignant process. Adv Cancer Res 71: 241-319.

41. Georgolios A, Batistatou A, Charalabopoulos A, Manolopoulos L Charalabopoulos K (2006) The role of CD44 adhesion molecule in oral cavity cancer. Exp Oncol 28: 94-98.

42. Bates RC, Edwards NS, Burns GF, Fisher DE (2001) A CD44 survival pathway triggers chemoresistance via lyn kinase and phosphoinositide 3-kinase/Akt in colon carcinoma cells. Cancer Res 61: 5275-5283.

43. Dontu G, Liu S, Wicha MS (2005) Stem cells in mammary development and carcinogenesis: implications for prevention and treatment Stem Cell Rev 1 : 207-213.

44. Reim F, Dombrowski Y, Ritter C, Buttmann M, Häusler S, et al. (2009) Immunoselection of breast and ovarian cancer cells with trastuzumab and natural killer cells: selective escape of CD44high/CD24low/HER2low breast cancer stem cells. Cancer Res 69: 8058-8066.

45. Lee CJ, Dosch J, Simeone DM (2008) Pancreatic cancer stem cells. J Clin Oncol 26: 2806-2812.

46. Ding Q, Chang CJ, Xie X, Xia W, Yang JY, et al. (2011) APOBEC3G promotes liver metastasis in an orthotopic mouse model of colorectal cancer and predicts human hepatic metastasis. J Clin Invest 121: 4526-4536.

47. Bellizzi A, Sebastian S, Ceglia P, Centonze M, Divella R, et al. (2013) Co expression of CD133(+)/CD44(+) in human colon cancer and liver metastasis. JCell Physiol 228: 408-415.

48. Horst D, Kriegl L, Engel J, Kirchner T, Jung A (2009) Prognostic significance of the cancer stem cell markers CD133, CD44, and CD166 in colorectal cancer Cancer Invest 27: 844-850.

49. Salnikov AV, Kusumawidjaja G, Rausch V, Bruns H, Gross W, et al. (2009) Cancer stem cell marker expression in hepatocellular carcinoma and liver metastases is not sufficient as single prognostic parameter. Cancer Lett 275 185-193.

50. Galizia G, Gemei M, Del Vecchio L, Zamboli A, Di Noto R, et al. (2012) Combined CD133/CD44 expression as a prognostic indicator of disease-free survival in patients with colorectal cancer. Arch Surg 147: 18-24.

51. Chen KL, Pan F, Jiang $H$, Chen JF, Pei L, et al. (2011) Highly enriched CD133(+)CD44(+) stem-like cells with CD133(+)CD44(high) metastatic subse in HCT116 colon cancer cells. Clin Exp Metastasis 28: 751-763.

52. Fang DD, Kim YJ, Lee CN, Aggarwal S, McKinnon K, et al. (2010) Expansion of CD133(+) colon cancer cultures retaining stem cell properties to enable cance stem cell target discovery. Br J Cancer 102: 1265-1275.

53. Steinert G, Schölch S, Koch M, Weitz J (2012) Biology and significance of circulating and disseminated tumour cells in colorectal cancer. Langenbecks Arch Surg 397: 535-542.

54. Pilati P, Mocellin S, Bertazza L, Galdi F, Briarava M, et al. (2012) Prognostic value of putative circulating cancer stem cells in patients undergoing hepatic resection for colorectal liver metastasis. Ann Surg Oncol 19: 402-408.

55. linuma H, Watanabe T, Mimori K, Adachi M, Hayashi N, et al. (2011) Clinica significance of circulating tumor cells, including cancer stem-like cells, in peripheral blood for recurrence and prognosis in patients with Dukes' stage B and C colorectal cancer. J Clin Oncol 29: 1547-1555

56. Tentes IK, Schmidt WM, Krupitza G, Steger GG, Mikulits W, et al. (2010) Longterm persistence of acquired resistance to 5 -fluorouracil in the colon cancer cell line SW620. Exp Cell Res 316: 3172-3181.

57. Fan X, Ouyang N, Teng H, Yao H (2011) Isolation and characterization of spheroid cells from the HT29 colon cancer cell line. Int J Colorectal Dis 26 : 1279-1285.

58. Dallas NA, Xia L, Fan F, Gray MJ, Gaur P, et al. (2009) Chemoresistant 
Citation: Bellizzi A, Tommasi S (2013) Cancer Cells with Stem Cell-Like Phenotypes and Liver Metastasis from Colon Carcinoma. J Liver 2: 114. doi:10.4172/2167-0889.1000114

colorectal cancer cells, the cancer stem cell phenotype, and increased sensitivity to insulin-like growth factor-I receptor inhibition. Cancer Res 69: 1951-1957.

59. Patel VB, Yu Y, Das JK, Patel BB, Majumdar AP (2009) Schlafen-3: a novel regulator of intestinal differentiation. Biochem Biophys Res Commun 388: 752756

60. Oh PS, Patel VB, Sanders MA, Kanwar SS, Yu Y, et al. (2011) Schlafen-3 decreases cancer stem cell marker expression and autocrine/juxtacrine signaling in FOLFOX-resistant colon cancer cells. Am J Physiol Gastrointest Liver Physiol 301: G347-355.

61. Song B, Wang Y, Xi Y, Kudo K, Bruheim S, et al. (2009) Mechanism of chemoresistance mediated by miR-140 in human osteosarcoma and colon cancer cells. Oncogene 28: 4065-4074.
62. Ojima I, Chen J, Sun L, Borella CP, Wang T, et al. (2008) Design, synthesis, and biological evaluation of new-generation taxoids. J Med Chem 51: 32033221.

63. Hwang WL, Yang MH, Tsai ML, Lan HY, Su SH, et al. (2011) SNAIL regulates interleukin-8 expression, stem cell-like activity, and tumorigenicity of human colorectal carcinoma cells. Gastroenterology 141: 279-291.

64. Wang C, Xie J, Guo J, Manning HC, Gore JC, et al. (2012) Evaluation of CD44 and CD133 as cancer stem cell markers for colorectal cancer. Oncol Rep 28 1301-1308.

65. Botchkina GI, Zuniga ES, Das M, Wang Y, Wang H, et al. (2010) Newgeneration taxoid SB-T-1214 inhibits stem cell-related gene expression in 3D cancer spheroids induced by purified colon tumor-initiating cells. Mol Cancer 9: 192. 